J. Nonlinear Var. Anal. 6 (2022), No. 1, pp. 37-50

Available online at http://jnva.biemdas.com

https://doi.org/10.23952/jnva.6.2022.1.03

\title{
THE VISCOSITY APPROXIMATION METHOD FOR ACCRETIVE OPERATORS IN BANACH SPACES
}

\author{
HONG-KUN XU ${ }^{1,2, *}$, NAJLA ALTWAIJRY ${ }^{3}$, IMTITHAL ALZUGHAIBI ${ }^{3}$, SOUHAIL CHEBBI $^{3}$ \\ ${ }^{1}$ School of Science, Hangzhou Dianzi University, Hangzhou 310018, China \\ ${ }^{2}$ College of Mathematics and Information Science, Henan Normal University, Xinxiang 453007, China \\ ${ }^{3}$ Department of Mathematics, College of Science, King Saud University, Riyadh 11451, Saudi Arabia
}

\begin{abstract}
We extend the viscosity approximation method (VAM) to accretive operators (via their resolvents) in a uniformly convex and/or uniformly Gâteaux differentiable Banach space $X$ to find a zero of an $m$-accretive operator and of the sum of two $m$-accretive operators. In all cases, we prove the strong convergence of our VAM algorithms and the limit of the iterates is identified as the unique sunny nonexpansive retraction onto to the zero set of the operator.
\end{abstract}

Keywords. Accretive operator; Banach space; Fixed point; Forward-backward splitting; viscosity approximation method.

\section{INTRODUCTION}

Let $X$ be a Banach space with norm $\|\cdot\|$, and let $A$ be an $m$-accretive operator in $X$ such that the set of zeros of $A$ is nonempty, $A^{-1} 0:=\{x \in D(A): 0 \in A x\} \neq \emptyset$. Finding a zero of $A$ is an important topic in the theory of accretive operators. One way to do this is via the resolvent $J_{\lambda}^{A}$ of $A$. Indeed, Reich [1, Theorem 1] proved that, for each $x \in X, J_{\lambda}^{A} x$ converges as $\lambda \rightarrow \infty$ to the point $Q x \in A^{-1} 0$, and $Q$ defines the unique sunny nonexpansive retraction from $X$ onto $A^{-1} 0$ if $X$ is uniformly smooth. Since the resolvent $J_{\lambda}^{A} x$ is also a nonexpansive mapping with fixed point set equal to $A^{-1} 0$ for each $\lambda>0$, fixed point methods such as Mann's [2] and Halpern's [3] (see [4] for a brief survey) methods have been used to find zeros of accretive operators [5, 6].

In a recent paper [7], Aoyama and Toyoda applied Halpern's method to the resolvent $J_{\lambda}^{A}$ to prove the following result (which was also proved in [5] in a uniformly smooth Banach space with a weakly continuous duality map $J_{\mu}$ for some gauge $\mu$ ).

Theorem 1.1. [7, Theorem 3.1, p. 809] Let E be a uniformly convex Banach space with a uniformly Gâteaux differentiable norm, let $C$ be a nonempty closed convex subset of $E$, and let $A \subset E \times E$ an accretive operator. Suppose $A^{-1} 0 \neq \emptyset$ and $\overline{D(A)} \subset C \subset R(I+\lambda A)$ for all $\lambda>0$. Assume $\left\{\alpha_{n}\right\} \subset(0,1]$ and $\left\{\lambda_{n}\right\}$ fulfil the conditions:

( $\alpha) \lim _{n \rightarrow \infty} \alpha_{n}=0$ and $\sum_{n=0}^{\infty} \alpha_{n}=\infty$;

\footnotetext{
${ }^{*}$ Corresponding author.

E-mail addresses: xuhk@hdu.edu.cn (H.K. Xu), najla@ksu.edu.sa (N. Altwaijry), 439204397@ student.ksu. edu.sa (I. Alzughaibi), schebbi@ksu.edu.sa (S. Chebbi).

Received July 5, 2021; Accepted September 3, 2021.
}

(C)2022 Journal of Nonlinear and Variational Analysis 
( $\lambda \inf _{n \geq 0} \lambda_{n}>0$.

Fix $u \in C$ and define a sequence $\left\{x_{n}\right\}$ by $x_{n+1}=\alpha_{n} u+\left(1-\alpha_{n}\right) J_{\lambda_{n}}^{A} x_{n}, n \geq 0$, where $x_{0} \in C$. Then $\left\{x_{n}\right\}$ converges strongly to $Q u$, where $Q$ is the unique sunny nonexpansive retraction of $C$ onto $A^{-1} 0$.

The viscosity approximation method (VAM) was introduced to the optimization theory by Attouch [8] and developed to nonexpansive mappings by Moudafi [9] in Hilbert spaces and Xu [10] in Banach spaces, respectively.

Let $X$ be a Banach space, $C$ a closed convex subset of $X$, and $T: C \rightarrow C$ a nonexpansive mapping with $\operatorname{Fix}(T) \neq \emptyset$. We use $\Pi_{C}$ to denote the collection of all contractions on $C$. Namely, $f \in \Pi_{C}$ means that $f: C \rightarrow C$ is a contraction: $\|f(x)-f(y)\| \leq \alpha\|x-y\|$ for $x, y \in C$ and some $\alpha \in[0,1)$.

The VAM for a nonexpansive mapping $T: C \rightarrow C$ is outlined as follows. For $t \in(0,1)$ and $f \in \Pi_{C}$, the map $x \mapsto t f(x)+(1-t) T x$ is a contraction from $C$ into $C$, and thus possesses a unique fixed point $x_{t} \in C$. That is,

$$
x_{t}=t f\left(x_{t}\right)+(1-t) T x_{t} .
$$

Theorem 1.2. [10, Theorem 4.1] Let $X$ be a uniformly smooth Banach space (uniform Gâteaux differentiability is sufficient), $C$ a closed convex subset of $X, T: C \rightarrow C$ a nonexpansive mapping with $\operatorname{Fix}(T) \neq \emptyset$, and $f \in \Pi_{C}$. Then $\left\{x_{t}\right\}$ defined by (1.1) converges strongly to a point in $F i x(T)$. If we define $Q: \Pi_{C} \rightarrow$ Fix $(T)$ by

$$
Q(f):=\lim _{t \rightarrow 0} x_{t}, \quad f \in \Pi_{C},
$$

then $Q(f)$ solves the variational inequality

$$
\langle(I-f) Q(f), J(Q(f)-p)\rangle \leq 0, \quad f \in \Pi_{C}, p \in F i x(T) .
$$

In particular, if $f=u \in C$ is a constant, then (1.2) is reduced to the sunny nonexpansive retraction of Reich from C onto Fix $(T)$

$$
\langle Q(u)-u, J(Q(u)-p)\rangle \leq 0, \quad u \in C, p \in F i x(T) .
$$

The purpose of this paper is to extend VAM to the resolvent $J_{\lambda}^{A}$ for finding a zero of an $m$-accretive operator $A$ in a Banach space. The VAM generates a sequence via the iteration process

$$
x_{n+1}=\alpha_{n} f\left(x_{n}\right)+\left(1-\alpha_{n}\right) J_{\lambda_{n}}^{A} x_{n}, \quad n=0,1, \cdots .
$$

We will investigate the strong convergence analysis of the VAM algorithm (1.3) in the framework of the underlying space $X$ being uniformly convex and/or uniformly Gâteaux differentiable. We will also introduce a split VAM (see Section 3 below) for solving the inclusion

$$
0 \in(A+B) x,
$$

where $A$ and $B$ are both $m$-accretive operators in a Banach space $X$.

The structure of the paper is as follows. In Section2, we introduce some preliminaries, including the concepts from Banach space theory such as uniform convexity, uniform smoothness, and duality maps, and nonlinear mappings such as nonexpansive mappings and accretive operators. Two useful lemmas on real sequences are included as well, which are helpful in proving the strong convergence of our algorithms in Sections 3 and 4. In Section 3, we prove the strong 
convergence of the VAM for finding a zero of the accretive operator $A$, and in Section 4, we extend the convergence result obtained in Section 3 to the composite case of finding a zero of the sum of two accretive operators $A+B$. Finally, a brief summary is given in Section 5 , the last section.

\section{PRELIMINARIES}

2.1. Convexity and smoothness. Let $(X,\|\cdot\|)$ be a Banach space with dual $X^{*}$ and let $S_{X}:=$ $\{x \in X:\|x\|=1\}$ be the unit sphere of $X$. Consider the

$$
\lim _{t \rightarrow 0} \frac{\|x+t y\|-\|x\|}{t}
$$

where $x, y \in S_{X}$. We say that (the norm of) $X$ is

- Gâteaux differentiable (or smooth) if limit (2.1) exists for each $x, y \in S_{X}$;

- uniformly Gâteaux differentiable if limit (2.1) exists for each $x, y \in S_{X}$, and is attained uniformly in $x \in S_{X}$;

- Fréchet differentiable if limit (2.1) exists for each $x, y \in S_{X}$ and is attained uniformly in $y \in S_{X}$

- uniformly smooth if limit (2.1) exists and is attained uniformly in both $x, y \in S_{X}$.

Recall that the modulus of convexity of $X$ is the function $\delta_{X}:(0,2] \rightarrow[0,1]$ defined by

$$
\delta_{X}(\varepsilon)=\inf \left\{1-\frac{\|x+y\|}{2}: x, y \in S_{X},\|x-y\| \geq \varepsilon\right\} .
$$

We say that $X$ is uniformly convex if $\delta_{X}(\varepsilon)>0$ for any $\varepsilon \in(0,2]$. We also say that $X$ is $p$ uniformly convex for some $p>1$ if there exists a constant $c_{p}>0$ such that $\delta_{X}(\varepsilon) \geq c_{p} \varepsilon^{p}$ for $\varepsilon \in(0,2]$.

It is known that if $X$ is uniformly convex, then, given sequences $\left\{x_{n}\right\}$ and $\left\{y_{n}\right\}$ in $X$ and real numbers $d>0$ and $c \in(0,1)$, the implication below holds:

$$
\lim _{n \rightarrow \infty}\left\|x_{n}\right\|=\lim _{n \rightarrow \infty}\left\|y_{n}\right\|=\lim _{n \rightarrow \infty}\left\|c x_{n}+(1-c) y_{n}\right\|=d \Rightarrow \lim _{n \rightarrow \infty}\left\|x_{n}-y_{n}\right\|=0 .
$$

Recall also that the modulus of smoothness of $X$ is defined as the function $\rho_{X}(\tau): \mathbb{R}_{+} \rightarrow \mathbb{R}_{+}$ given by

$$
\rho_{X}(\tau)=\sup \left\{\frac{\|x+\tau y\|+\|x-\tau y\|}{2}-1: x, y \in S_{X}\right\} .
$$

It is known that $X$ is uniformly smooth if and only if $\lim _{\tau \rightarrow 0} \rho_{X}(\tau) / \tau=0$. Moreover, we say that $X$ is $q$-uniformly smooth for some $1<q \leq 2$ if there is a constant $c_{q}>0$ with the property $\rho_{X}(\tau) \leq c_{q} \tau^{q}$ for $\tau>0$. It is known that $X$ is $p$-uniformly convex if and only if $X^{*}$ is $q$-uniformly smooth, with $q=p /(p-1)$. For example, $L^{p}$ is 2 -uniformly convex and $p$-uniformly smooth if $1<p \leq 2$, and $p$-uniformly convex and 2-uniformly smooth if $2 \leq p<\infty$. For more details on the convexity and smoothness of Banach spaces, we refer to [11].

2.2. Duality maps. The concept of general duality maps was introduced to nonlinear mappings in a series of articles by Browder [12]-[16]. For the purpose of this paper, we need the generalized duality map $J_{p}$ of $X$ for $p \in(1 . \infty)$, defined by

$$
J_{p}(x)=\left\{x^{*} \in X^{*}:\left\langle x, x^{*}\right\rangle=\|x\|^{p},\left\|x^{*}\right\|=\|x\|^{p-1}\right\}, \quad x \in X .
$$

We write $J$ for $J_{2}$ and call it the normalized duality map of $X$. 
It is worth pointing out that the duality maps can be used to characterize different sorts of smoothness. Indeed, we will use the following facts.

- $X$ is uniformly Gâteaux differentiable if and only if the normalized duality map $J$ of $X$ is norm-to-weak ${ }^{*}$ uniformly continuous on bounded sets of $X$;

- $X$ is uniformly smooth if and only if the normalized duality map $J$ of $X$ is norm-to-norm uniformly continuous on bounded sets of $X$.

In a $q$-uniformly smooth Banach space, the generalized duality $J_{q}$ is more appropriate in [17] than the normalized duality map $J$, due to the following lemma.

Lemma 2.1. [18] If a Banach space $X$ is q-uniformly smooth for some $q \in$ (1.2], then there exists a constant $\kappa_{q}>0$ such that

$$
\|x+y\|^{q} \leq\|x\|^{q}+q\left\langle y, J_{q}(x)\right\rangle+\kappa_{q}\|y\|^{q}, \quad x, y \in X .
$$

Remark. If $X=L^{p}$, then, for $2 \leq p<\infty, X$ is 2-uniformly smooth with $\kappa_{2}=p-1$; and for $1<p<2, X$ is $p$-uniformly smooth with $\kappa_{p}=\left(1+\tau_{p}^{p-1}\right)\left(1+\tau_{p}\right)^{1-p}$, where $\tau_{p} \in(0,1)$ is the unique solution of the equation $(p-2) \tau^{p-1}+(p-1) \tau^{p-2}-1=0$ for $0<\tau<1$.

2.3. Nonexpansive mappings and accretive operators. Let $X$ be a Banach space with dual $X^{*}$ and let $C$ be a nonempty closed convex subset of $X$. A mapping $T: C \rightarrow X$ is said to be nonexpansive if

$$
\|T x-T y\| \leq\|x-y\|, \quad x, y \in C .
$$

We use $\operatorname{Fix}(T)$ to denote the set of fixed points of $T$; namely, $\operatorname{Fix}(T)=\{x \in C: T x=x\}$.

Let $A$ be a mapping with domain $D(A)$ and range $R(A)$ in $X$. For the sake of convenience, we shall identify $A$ with its graph $G(A):=\{(x, y) \in X \times X: x \in D(A), y \in A x\}$ in the product space $X \times X$. We say that $A$ is accretive ([19]) if, for $\left(x_{i}, y_{i}\right) \in A(i=1,2)$, there is $j \in J\left(x_{2}-x_{1}\right)$ such that

$$
\left\langle y_{2}-y_{1}, j\right\rangle \geq 0 \text {. }
$$

It is known [20] that the accretiveness of $A$ is equivalent to

$$
\left\|x_{2}-x_{1}\right\| \leq\left\|x_{2}-x_{1}+c\left(y_{2}-y_{1}\right)\right\| \quad \text { for }\left(x_{i}, y_{i}\right) \in A(i=1,2) \text { and } c>0 .
$$

An accretive operator $A$ is said (i) to satisfy the range condition if $R(I+\lambda A) \supset \overline{D(A)}$ for all $\lambda>0$, and (ii) to be $m$-accretive if $R(I+\lambda A)=X$ for all $\lambda>0$. If $A$ is accretive and $\lambda>0$, then $J_{\lambda}^{A}:=(I+\lambda A)^{-1}$ denotes the resolvent of $A$. It is known that $J_{\lambda}^{A}$ is single-valued and nonexpansive from $R(I+\lambda A)$ to $D(A)$. Moreover, $\operatorname{Fix}\left(J_{\lambda}^{A}\right)=A^{-1} 0$ for all $\lambda>0$. The relation below is referred to as the resolvent identity

$$
J_{\lambda} x=J_{\gamma}\left(\frac{\gamma}{\lambda} x+\left(1-\frac{\gamma}{\lambda}\right) J_{\lambda} x\right)
$$

for all $x \in X$ and $\lambda, \gamma>0$.

Given $v>0$ and $q \in(1, \infty)$, we say that an accretive operator $A$ is $v$-inverse strongly accretive ( $v$-isa) of order $q[21]$ if, for each $x, y \in \mathscr{D}(A)$, there exists $j_{q} \in J_{q}(x-y)$ such that

$$
\left\langle u-v, j_{q}\right\rangle \geq v\|u-v\|^{q}, \quad(x, u) \in A,(y, v) \in A .
$$

When $q=2$, we simply say that $A$ is $v$-isa. This is a Banach space extension of the notion of strongly inverse monotone operators in a Hilbert space.

For more details of accretive operators, the reader is referred to [20, 22]. 
2.4. Tools for convergence of real sequences. The following convergence results on sequences of real numbers play a key role in the strong convergence analysis of VAM (1.3).

Lemma 2.2. ([23, Lemma 2.5]) Assume that $\left(s_{n}\right)$ is a sequence of nonnegative real numbers satisfying the condition:

$$
s_{n+1} \leq\left(1-\lambda_{n}\right) s_{n}+\lambda_{n} \mu_{n}+\sigma_{n}, \quad n \geq 0,
$$

where $\left(\lambda_{n}\right)$ and $\left(\sigma_{n}\right)$ are sequences in $(0,1)$, and $\left(\beta_{n}\right)$ is a sequence in $\mathbb{R}$. Assume that

(i) $\sum_{n=1}^{\infty} \lambda_{n}=\infty$,

(ii) $\limsup _{n \rightarrow \infty} \mu_{n} \leq 0$, and

(iii) $\sum_{n=1}^{\infty} \sigma_{n}<\infty$.

Then $\lim _{n \rightarrow \infty} s_{n}=0$.

Lemma 2.3. [24, Lemma 2.6] Let $\left\{s_{n}\right\}$ be a sequence of nonnegative real numbers satisfying the condition:

$$
s_{n+1} \leq\left(1-\lambda_{n}\right) s_{n}+\lambda_{n} \mu_{n}, \quad n \geq 0 .
$$

Suppose that $\left\{\lambda_{n}\right\}$ is a sequence in $(0,1)$ satisfying the condition (i) of Lemma 2.2, and $\left\{\mu_{n}\right\}$ is a sequence of real numbers satisfying the condition (for any subsequence $\left\{n_{k}\right\}$ of positive integers):

$$
\limsup _{k \rightarrow \infty} \mu_{n_{k}} \leq 0 \quad \text { whenever } \quad \limsup _{k \rightarrow \infty}\left(s_{n_{k}+1}-s_{n_{k}}\right) \geq 0 .
$$

Then $\lim _{n \rightarrow \infty} s_{n}=0$.

\section{Viscosity ApProximation Method}

We begin with a technical result related to the unique sunny nonexpansive retraction $Q$ as constructed in Theorem 1.2. This lemma plays a key role in proving the strong convergence of our VAM algorithms, due to the arbitrariness of the approximating fixed point of the nonexpansive mapping $T$.

Lemma 3.1. Let the assumptions of Theorem 1.2 hold and suppose that $\left\{z_{n}\right\}$ is an arbitrary approximating fixed point of $T$ (i.e., $\lim _{n \rightarrow \infty}\left\|z_{n}-T z_{n}\right\|=0$ ). Then

$$
\limsup _{n \rightarrow \infty}\left\langle Q(f)-f(Q(f)), J\left(Q(f)-z_{n}\right)\right\rangle \leq 0 .
$$

Proof. Eq. (3.1) is [10, Eq. (39), p. 289] whose proof is still valid for the case that $\left\{x_{n}\right\}$ is replaced with any sequence $\left\{z_{n}\right\}$ such that $\left\|z_{n}-T z_{n}\right\| \rightarrow 0$. The special case that $f(x) \equiv u$ was also proved in [7, Lemma 2.9].

Lemma 3.2. Let $X$ be a uniformly convex Banach space and let $A$ be an accretive operator satisfying the range condition and with $A^{-1} 0 \neq \emptyset$. Then, for any sequence $\left\{\lambda_{n}\right\}$ of positive real numbers, and bounded sequences $\left\{x_{n}\right\}$ and $\left\{y_{n}\right\}$ in $\overline{D(A)}$ such that $\left\|x_{n}-y_{n}\right\|-\| J_{\lambda_{n}}^{A} x_{n}-$ $J_{\lambda_{n}}^{A} y_{n}\|\rightarrow 0\|,\left(x_{n}-y_{n}\right)-\left(J_{\lambda_{n}}^{A} x_{n}-J_{\lambda_{n}}^{A} y_{n}\right) \| \rightarrow 0$. In particular, if $z \in A^{-1} 0$ is such that $\left\|x_{n}-z\right\|-$ $\left\|J_{\lambda_{n}}^{A} x_{n}-z\right\| \rightarrow 0$, then $\left\|x_{n}-J_{\lambda_{n}}^{A} x_{n}\right\| \rightarrow 0$.

Proof. Since each resolvent of accretive operators is firmly nonexpansive [25], one has

$$
\left\|J_{\lambda}^{A} x-J_{\lambda}^{A} y\right\| \leq\left\|c(x-y)+(1-c)\left(J_{\lambda}^{A} x-J_{\lambda}^{A} y\right)\right\|, \quad x, y \in \overline{D(A)}, c \in[0,1] .
$$


It turns out that

$$
\left\|J_{\lambda_{n}}^{A} x_{n}-J_{\lambda_{n}}^{A} y_{n}\right\| \leq\left\|c\left(x_{n}-y_{n}\right)+(1-c)\left(J_{\lambda_{n}}^{A} x_{n}-J_{\lambda_{n}}^{A} y_{n}\right)\right\|, \quad c \in[0,1]
$$

We may assume $\left\|x_{n}-y_{n}\right\| \rightarrow d>0$. Thus $\left\|J_{\lambda_{n}}^{A} x_{n}-J_{\lambda_{n}}^{A} y_{n}\right\| \rightarrow d$. Moreover, it follows from (3.2) that $\left\|c\left(x_{n}-y_{n}\right)+(1-c)\left(J_{\lambda_{n}}^{A} x_{n}-J_{\lambda_{n}}^{A} y_{n}\right)\right\| \rightarrow d$ for $c \in(0,1)$. Then the uniform convexity of $X$ asserts that $\left\|\left(x_{n}-y_{n}\right)-\left(J_{\lambda_{n}}^{A} x_{n}-J_{\lambda_{n}}^{A} y_{n}\right)\right\| \rightarrow 0$ as $n \rightarrow \infty$.

Theorem 3.1. Let $X$ be a uniformly convex Banach space with a uniformly Gâteaux differentiable norm and let $A \subset X \times X$ be an accretive operator in $X$ with $A^{-1} 0 \neq \emptyset$. Suppose that $C$ is a nonempty closed convex subset of $X$ such that $\overline{D(A)} \subset C \subset R(I+\lambda A)$ for all $\lambda>0$. Let $f: C \rightarrow C$ be an $\alpha$-contraction with $\alpha \in[0,1)$. Define a sequence $\left\{x_{n}\right\}$ by the viscosity approximation method (VAM)

$$
x_{n+1}=\alpha_{n} f\left(x_{n}\right)+\left(1-\alpha_{n}\right) J_{\lambda_{n}}^{A} x_{n}, \quad n \geq 0
$$

where $x_{0} \in C$ is an initial guess. Assume that $\left\{\alpha_{n}\right\} \subset(0,1]$ and $\left\{\lambda_{n}\right\} \subset \mathbb{R}$ fulfil the conditions:

( $\alpha) \lim _{n \rightarrow \infty} \alpha_{n}=0$ and $\sum_{n=0}^{\infty} \alpha_{n}=\infty$;

( $\lambda) \inf _{n \geq 0} \lambda_{n}>0$.

Then $\left\{x_{n}\right\}$ converges strongly to $Q(f)$, where $Q$ is the unique sunny nonexpansive retraction of $\Pi_{C}$ onto $A^{-1} 0$ as defined in (1.2).

Proof. First we show that the sequence $\left\{x_{n}\right\}$ is bounded. Indeed, take $z \in A^{-1} 0$ to deduce from (3.3) that (notice $z \in F i x\left(J_{\lambda}^{A}\right)$ for all $\lambda>0$ )

$$
\begin{aligned}
\left\|x_{n+1}-z\right\| & =\left\|\alpha_{n}\left[f\left(x_{n}\right)-z\right]+\left(1-\alpha_{n}\right)\left[J_{\lambda_{n}}^{A} x_{n}-z\right]\right\| \\
& \leq \alpha_{n}\left\|f\left(x_{n}\right)-z\right\|+\left(1-\alpha_{n}\right)\left\|J_{\lambda_{n}}^{A} x_{n}-z\right\| \\
& \leq \alpha_{n}\left(\left\|f\left(x_{n}\right)-f(z)\right\|+\|f(z)-z\|\right)+\left(1-\alpha_{n}\right)\left\|x_{n}-z\right\| \\
& \leq \alpha_{n}\left(\alpha\left\|x_{n}-z\right\|+\|f(z)-z\|\right)+\left(1-\alpha_{n}\right)\left\|x_{n}-z\right\| \\
& =\left(1-(1-\alpha) \alpha_{n}\right)\left\|x_{n}-z\right\|+\alpha_{n}\|f(z)-z\| \\
& \leq \max \left\{\left\|x_{n}-z\right\|,\|f(z)-z\| /(1-\alpha)\right\} .
\end{aligned}
$$

By induction, we obtain

$$
\left\|x_{n}-z\right\| \leq \max \left\{\left\|x_{0}-z\right\|,\|f(z)-z\| /(1-\alpha)\right\}
$$

for all $n \geq 0$. Consequently, $\left\{x_{n}\right\}$ is bounded, so are $\left\{f\left(x_{n}\right)\right\}$ and $\left\{J_{\lambda_{n}}^{A} x_{n}\right\}$. Fix a constant $M$ such that

$$
M \geq \max \left\{\left\|J_{\lambda_{n}}^{A} x_{n}-x_{n}\right\|,\left\|J_{\lambda_{n}}^{A} x_{n}-f\left(x_{n}\right)\right\|\right\}, \quad n \geq 0 .
$$

It then follows from (3.3) that

$$
\left\|x_{n+1}-J_{\lambda_{n}}^{A} x_{n}\right\|=\alpha_{n}\left\|f\left(x_{n}\right)-J_{\lambda_{n}}^{A} x_{n}\right\| \leq M \alpha_{n} \rightarrow 0
$$


Put $\tilde{x}=Q(f)$. We then deduce again from (3.3) that

$$
\begin{aligned}
\left\|x_{n+1}-\tilde{x}\right\|^{2}= & \left\|\alpha_{n}\left[f\left(x_{n}\right)-\tilde{x}\right]+\left(1-\alpha_{n}\right)\left[J_{\lambda_{n}}^{A} x_{n}-\tilde{x}\right]\right\|^{2} \\
\leq & \left\|\left(1-\alpha_{n}\right)\left[J_{\lambda_{n}}^{A} x_{n}-\tilde{x}\right]\right\|^{2}+2 \alpha_{n}\left\langle f\left(x_{n}\right)-\tilde{x}, J\left(x_{n+1}-\tilde{x}\right)\right\rangle \\
\leq & \left(1-\alpha_{n}\right)^{2}\left\|x_{n}-\tilde{x}\right\|^{2}+2 \alpha_{n}\left\langle f\left(x_{n}\right)-f(\tilde{x}), J\left(x_{n+1}-\tilde{x}\right)\right\rangle \\
& +2 \alpha_{n}\left\langle f(\tilde{x})-\tilde{x}, J\left(x_{n+1}-\tilde{x}\right)\right\rangle \\
\leq & \left(1-\alpha_{n}\right)^{2}\left\|x_{n}-\tilde{x}\right\|^{2}+2 \alpha_{n} \alpha\left\|x_{n}-\tilde{x}\right\| \cdot\left\|x_{n+1}-\tilde{x}\right\| \\
& +2 \alpha_{n}\left\langle f(\tilde{x})-\tilde{x}, J\left(x_{n+1}-\tilde{x}\right)\right\rangle \\
\leq & \left(1-\alpha_{n}\right)^{2}\left\|x_{n}-\tilde{x}\right\|^{2}+\alpha_{n} \alpha\left(\left\|x_{n}-\tilde{x}\right\|^{2}+\left\|x_{n+1}-\tilde{x}\right\|^{2}\right) \\
& +2 \alpha_{n}\left\langle f(\tilde{x})-\tilde{x}, J\left(x_{n+1}-\tilde{x}\right)\right\rangle \\
= & \left(1-(2-\alpha) \alpha_{n}+\alpha_{n}^{2}\right)\left\|x_{n}-\tilde{x}\right\|^{2}+\alpha_{n} \alpha\left\|x_{n+1}-\tilde{x}\right\|^{2} \\
& +2 \alpha_{n}\left\langle f(\tilde{x})-\tilde{x}, J\left(x_{n+1}-\tilde{x}\right)\right\rangle .
\end{aligned}
$$

It turns out that

$$
\left\|x_{n+1}-\tilde{x}\right\|^{2} \leq \frac{1-(2-\alpha) \alpha_{n}+\alpha_{n}^{2}}{1-\alpha \alpha_{n}}\left\|x_{n}-\tilde{x}\right\|^{2}+\frac{2 \alpha_{n}}{1-\alpha \alpha_{n}}\left\langle f(\tilde{x})-\tilde{x}, J\left(x_{n+1}-\tilde{x}\right)\right\rangle .
$$

This can be rewritten as

$$
s_{n+1} \leq\left(1-\beta_{n}\right) s_{n}+\beta_{n} \gamma_{n}
$$

where $s_{n}=\left\|x_{n}-\tilde{x}\right\|^{2}, \beta_{n}=\frac{2(1-\alpha) \alpha_{n}-\alpha_{n}^{2}}{1-\alpha \alpha_{n}} \rightarrow 0$ (as $\left.\alpha_{n} \rightarrow 0\right)$, and

$$
\gamma_{n}=\frac{2}{2(1-\alpha)-\alpha_{n}}\left\langle f(\tilde{x})-\tilde{x}, J\left(x_{n+1}-\tilde{x}\right)\right\rangle .
$$

Since $\alpha_{n} \rightarrow 0$, we easily find that $\beta_{n} / \alpha_{n} \rightarrow 2(1-\alpha)>0$. Hence, we obtain that $\sum_{n=0}^{\infty} \beta_{n}=\infty$ by virtue of condition $(\alpha)$. Also keep in mind that $\left\{\gamma_{n}\right\}$ is bounded.

We apply Lemma 2.3 to prove that $s_{n} \rightarrow 0$. Towards this, we assume that $\left\{s_{n_{k}}\right\}$ is a subsequence of $\left\{s_{n}\right\}$ such that $\liminf _{k \rightarrow \infty}\left(s_{n_{k}+1}-s_{n_{k}}\right) \geq 0$ (all we need to verify is $\limsup _{k \rightarrow \infty} \gamma_{n_{k}} \leq$ $0)$. From (3.8), and $\limsup _{n \rightarrow \infty}\left(s_{n_{k}+1}-s_{n_{k}}\right) \leq \limsup _{n \rightarrow \infty} \beta_{n_{k}} \gamma_{n_{k}}=0\left(\beta_{n} \rightarrow 0\right.$ and $\left\{\gamma_{n}\right\}$ is bounded), we must have $\lim _{k \rightarrow \infty}\left(s_{n_{k}+1}-s_{n_{k}}\right)=0$. Setting $\xi_{k}:=s_{n_{k}+1}-s_{n_{k}}$, we have

$$
\begin{aligned}
\left\|J_{\lambda_{n_{k}}}^{A} x_{n_{k}}-\tilde{x}\right\|^{2} & \leq\left\|x_{n_{k}}-\tilde{x}\right\|^{2}=s_{n_{k}}=s_{n_{k}+1}-\xi_{k}=\left\|x_{n_{k}+1}-\tilde{x}\right\|^{2}-\xi_{k} \\
& =\left\|\alpha_{n_{k}}\left(f\left(x_{n_{k}}\right)-\tilde{x}\right)+\left(1-\alpha_{n_{k}}\right)\left(J_{\lambda_{n_{k}}}^{A} x_{n_{k}}-\tilde{x}\right)\right\|^{2}-\xi_{k} \\
& \leq \alpha_{n_{k}}\left\|f\left(x_{n_{k}}\right)-\tilde{x}\right\|^{2}+\left(1-\alpha_{n_{k}}\right)\left\|J_{\lambda_{n_{k}}}^{A} x_{n_{k}}-\tilde{x}\right\|^{2}-\xi_{k} \\
& \leq \alpha_{n_{k}}\left\|f\left(x_{n_{k}}\right)-\tilde{x}\right\|^{2}+\left\|J_{\lambda_{n_{k}}}^{A} x_{n_{k}}-\tilde{x}\right\|^{2}-\xi_{k} .
\end{aligned}
$$

Consequently, we obtain that

$$
0 \leq\left\|x_{n_{k}}-\tilde{x}\right\|^{2}-\left\|J_{\lambda_{n_{k}}}^{A} x_{n_{k}}-\tilde{x}\right\|^{2} \leq \alpha_{n_{k}}\left\|f\left(x_{n_{k}}\right)-\tilde{x}\right\|^{2}-\xi_{k} \rightarrow 0 .
$$

It follows that

$$
\left\|x_{n_{k}}-\tilde{x}\right\|-\left\|J_{\lambda_{n_{k}}}^{A} x_{n_{k}}-\tilde{x}\right\| \rightarrow 0 .
$$

From Lemma 3.2, we conclude that

$$
\left\|x_{n_{k}}-J_{\lambda_{n_{k}}}^{A} x_{n_{k}}\right\| \rightarrow 0
$$


and

$$
\begin{aligned}
\left\|x_{n_{k}+1}-x_{n_{k}}\right\| & \leq\left\|x_{n_{k}+1}-J_{\lambda_{n_{k}}}^{A} x_{n_{k}}\right\|+\left\|x_{n_{k}}-J_{\lambda_{n_{k}}}^{A} x_{n_{k}}\right\| \\
& =\alpha_{n_{k}}\left\|f\left(x_{n_{k}}\right)-J_{\lambda_{n_{k}}}^{A} x_{n_{k}}\right\|+\left\|x_{n_{k}}-J_{\lambda_{n_{k}}}^{A} x_{n_{k}}\right\| \\
& \leq M \alpha_{n_{k}}+\left\|x_{n_{k}}-J_{\lambda_{n_{k}}}^{A} x_{n_{k}}\right\| \rightarrow 0 .
\end{aligned}
$$

Now, we set $\underline{\lambda}:=\inf _{n \geq 0} \lambda_{n}>0$, and $T:=J_{\underline{\lambda}}^{A}$. Then $T$ is nonexpansive and $\operatorname{Fix}(T)=A^{-1} 0$. Moreover, since

$$
\left\|x_{n_{k}}-T x_{n_{k}}\right\|=\left\|x_{n_{k}}-J_{\underline{\lambda}}^{A} x_{n_{k}}\right\| \leq 2\left\|x_{n_{k}}-J_{\lambda_{n_{k}}}^{A} x_{n_{k}}\right\| \rightarrow 0
$$

we find that $\left\{x_{n_{k}}\right\}$ is an approximating sequence of $T$. Consequently, we may apply Lemma 3.1 to obtain

$$
\limsup _{n \rightarrow \infty}\left\langle f(\tilde{x})-\tilde{x}, J\left(x_{n_{k}}-\tilde{x}\right)\right\rangle \leq 0 .
$$

However, since $\left\|x_{n_{k}+1}-x_{n_{k}}\right\| \rightarrow 0$, we must also have $\limsup _{n \rightarrow \infty}\left\langle f(\tilde{x})-\tilde{x}, J\left(x_{n_{k}+1}-\tilde{x}\right)\right\rangle \leq 0$. That is, $\limsup _{k \rightarrow \infty} \gamma_{n_{k}} \leq 0$. So, Lemma 2.3 is applicable to Eq. (3.8) and we assert that $s_{n} \rightarrow 0$, namely, $x_{n} \rightarrow \tilde{x}$ in norm. The proof is complete.

Remark 3.1. Theorem 3.1 extends [7, Theorem 3.1] to the viscosity approximation method for accretive operators in Banach spaces. In addition, the proof we provided here is simpler and more direct than the proof of [7, Theorem 3.1] that the authors employed Maingé's technical lemma (see [26, Lemma 3.1] that is not stated here).

We next show that if we choose parameters $\left\{\alpha_{n}\right\}$ and $\left\{\lambda_{n}\right\}$ appropriately, then the uniform convexity assumption in Theorem 3.1 can be removed.

Theorem 3.2. Let $X$ be a Banach space with a uniformly Gâteaux differentiable norm and let $A \subset X \times X$ be an accretive operator such that $A^{-1} 0 \neq \emptyset$. Suppose that $C$ is a nonempty closed convex subset of $X$ such that $\overline{D(A)} \subset C \subset R(I+\lambda A)$ for all $\lambda>0$. Assume that $f \in \Pi_{C}$ with contraction coefficient $\alpha \in[0,1)$ and the following conditions are satisfied:

(C1) $\lim _{n \rightarrow \infty} \alpha_{n}=0, \sum_{n=0}^{\infty} \alpha_{n}=\infty$, and $\sum_{n=0}^{\infty}\left|\alpha_{n+1}-\alpha_{n}\right|<\infty$;

(C2) either $\sum_{n=0}^{\infty}\left|\lambda_{n+1}-\lambda_{n}\right|<\infty$ or $\lim _{n \rightarrow \infty} \frac{\lambda_{n}}{\lambda_{n+1}}=1$;

(C3) $\inf _{n \geq 0} \lambda_{n}=: \underline{\lambda}>0$.

Then the sequence $\left\{x_{n}\right\}$ defined by VAM (3.3) converges strongly to $Q(f) \in A^{-1} 0$.

Proof. Let $Q: \Pi_{C} \rightarrow A^{-1} 0$ be the sunny nonexpansive retraction defined by (1.2), and set $\tilde{x}=$ $Q(f)$. From the first part of the proof of Theorem 3.1, we have the following:

- $\left\{x_{n}\right\}$ is bounded. Indeed (3.5) remains valid for $z \in A^{-1} 0$;

- the relation (3.8) remains true with $\gamma_{n}$, which is given by (3.9).

We will employ Lemma 2.2 to prove the strong convergence to $\tilde{x}$ of $\left\{x_{n}\right\}$. By (3.8), it remains to verify that $\limsup _{n \rightarrow \infty} \gamma_{n} \leq 0$. Namely,

$$
\limsup _{n \rightarrow \infty}\left\langle f(\tilde{x})-\tilde{x}, J\left(x_{n}-\tilde{x}\right)\right\rangle \leq 0 .
$$


Utilizing the definition of VAM (3.3) together with some manipulations, we obtain

$$
\begin{aligned}
x_{n+1}-x_{n}= & \alpha_{n}\left[f\left(x_{n}\right)-f\left(x_{n-1}\right)\right]+\left(\alpha_{n}-\alpha_{n-1}\right)\left[f\left(x_{n-1}\right)-J_{\lambda_{n-1}}^{A} x_{n-1}\right] \\
& +\left(1-\alpha_{n}\right)\left(J_{\lambda_{n}}^{A} x_{n}-J_{\lambda_{n}}^{A} x_{n-1}\right)+\left(1-\alpha_{n}\right)\left(J_{\lambda_{n}}^{A} x_{n-1}-J_{\lambda_{n-1}}^{A} x_{n-1}\right) .
\end{aligned}
$$

Since $f$ is an $\alpha$-contraction, we have $\left\|f\left(x_{n}\right)-f\left(x_{n-1}\right)\right\| \leq \alpha\left\|x_{n}-x_{n-1}\right\|$. By the resolvent identity, we derive that

$$
\begin{aligned}
\left\|J_{\lambda_{n}}^{A} x_{n-1}-J_{\lambda_{n-1}}^{A} x_{n-1}\right\| & =\left\|J_{\lambda_{n-1}}^{A}\left(\frac{\lambda_{n-1}}{\lambda_{n}} x_{n-1}+\left(1-\frac{\lambda_{n-1}}{\lambda_{n}}\right) J_{\lambda_{n}}^{A} x_{n-1}\right)-J_{\lambda_{n-1}}^{A} x_{n-1}\right\| \\
& \leq\left|1-\frac{\lambda_{n-1}}{\lambda_{n}}\right|\left|J_{\lambda_{n-1}}^{A} x_{n-1}-x_{n-1} \| \leq M\right| 1-\frac{\lambda_{n-1}}{\lambda_{n}} \mid .
\end{aligned}
$$

It follows from (3.12) that

$$
\left\|x_{n+1}-x_{n}\right\| \leq\left(1-(1-\alpha) \alpha_{n}\right)\left\|x_{n}-x_{n-1}\right\|+M\left(\left|\alpha_{n}-\alpha_{n-1}\right|+\left|1-\frac{\lambda_{n-1}}{\lambda_{n}}\right|\right) .
$$

Under the conditions (C1)-(C3), Lemma 2.2 is applicable to (3.13). Hence, we obtain $\| x_{n+1}-$ $x_{n} \| \rightarrow 0$. Then we have

$$
\left\|x_{n}-J_{\lambda_{n}}^{A} x_{n}\right\| \leq\left\|x_{n}-x_{n+1}\right\|+\left\|x_{n+1}-J_{\lambda_{n}}^{A} x_{n}\right\| \leq\left\|x_{n}-x_{n+1}\right\|+M \alpha_{n} \rightarrow 0 .
$$

Again, we set $T:=J_{\underline{\lambda}}^{A}$. Then $T$ is nonexpansive and $\operatorname{Fix}(T)=A^{-1} 0$. Moreover, since

$$
\left\|x_{n}-T x_{n}\right\|=\left\|x_{n}-J_{\underline{\lambda}}^{A} x_{n}\right\| \leq 2\left\|x_{n}-J_{\lambda_{n}}^{A} x_{n}\right\| \rightarrow 0
$$

namely, $\left\{x_{n}\right\}$ is an approximating fixed point of $T$, we can apply Lemma 3.1, with $\left\{z_{n}\right\}$ replaced with $\left\{x_{n}\right\}$, to conclude (3.1). In other words, (3.11) holds. This completes the proof.

\section{Split Viscosity Approximation Method}

In this section, we extend the VAM (3.3) to the composite case where the task is to find a zero of the sum of two accretive operators, namely, the inclusion:

$$
0 \in(A+B) x
$$

where $A$ and $B$ are $m$-accretive operators in a Banach space $X$ such that $A+B$ is also $m$-accretive. Assume that $S:=(A+B)^{-1} 0$ is nonempty, and let $T_{\lambda}:=J_{\lambda}^{B}(I-\lambda A)$ for $\lambda>0$.

Note that, in the Hilbert space setting, $A$ and $B$ are maximal monotone operators and inclusion (4.1) was considered in [27, 28] by using the forward-backward splitting method. A generalization to a Banach space setting was given in [21]. We will further extend the result of [21] to the viscosity approximation method.

Lemma 4.1. [21] We have

- $\operatorname{Fix}\left(T_{\lambda}\right)=(A+B)^{-1} 0$ for $\lambda>0$,

- $\left\|x-T_{\lambda} x\right\| \leq 2\left\|x-T_{\gamma} x\right\|$ for $x \in X$ and $0<\lambda<\gamma<\infty$.

The result below is [21, Theorem 3.7] without errors. 
Theorem 4.1. [21, Theorem 3.7] Let $X$ be a uniformly convex and q-uniformly smooth Banach space. Let $A: X \rightarrow X$ be an $v$-isa of order $q \in(1,2]$ and $B \subset X \times X$ be an $m$-accretive operator in $X$. We define a sequence $\left\{x_{n}\right\}$ by the iterative scheme

$$
x_{n+1}=\alpha_{n} u+\left(1-\alpha_{n}\right) J_{\lambda_{n}}^{B}\left(x_{n}-\lambda_{n} A x_{n}\right),
$$

where $u \in X,\left\{\alpha_{n}\right\} \subset(0,1]$, and $\left\{\lambda_{n}\right\} \subset(0,+\infty)$. Assume that the following conditions are satisfied:

(i) $\lim _{n \rightarrow \infty} \alpha_{n}=0, \sum_{n=0}^{\infty} \alpha_{n}=\infty$;

(ii) $0<\liminf _{n \rightarrow \infty} \lambda_{n} \leq \limsup \sup _{n \rightarrow \infty} \lambda_{n}<\left(v q / \kappa_{q}\right)^{1 /(q-1)}$.

Then $\left\{x_{n}\right\}$ converges in norm to $Q(u)$, where $Q$ is the sunny nonexpansive retraction from $X$ onto $S$.

We next study the VAM for inclusion (4.1) via the iteration process:

$$
x_{n+1}=\alpha_{n} f\left(x_{n}\right)+\left(1-\alpha_{n}\right) J_{\lambda_{n}}^{B}\left(x_{n}-\lambda_{n} A x_{n}\right),
$$

where $f: X \rightarrow X$ is an $\alpha$-contraction for some $\alpha \in[0,1)$.

Lemma 4.2. [21, Lemma 3.3] Let $X$ be a uniformly convex and q-uniformly smooth Banach space for some $q \in(1,2]$. Assume that $A$ is a single-valued $v$-isa in $X$ of order $q$ for some $v>0$. Let $\tau>0$. Then there exists a continuous, strictly increasing, and convex function $\phi: \mathbb{R}^{+} \rightarrow \mathbb{R}^{+}$with $\phi(0)=0$ (which may depend on $q, s$ ) such that, for all $x, y \in X$ with $\|x\| \leq \tau$ and $\|y\| \leq \tau$ and $\lambda>0$,

$$
\begin{aligned}
\left\|T_{\lambda} x-T_{\lambda} y\right\|^{q} \leq & \|x-y\|^{q}-\lambda\left(v q-\lambda^{q-1} \kappa_{q}\right)\|A x-A y\|^{q} \\
& -\phi\left(\left\|\left(I-J_{\lambda}\right)(I-\lambda A) x-\left(I-J_{\lambda}\right)(I-\lambda A) y\right\|\right),
\end{aligned}
$$

where $\kappa_{q}>0$ is the q-uniform smoothness coefficient of $X$ (see [21, Lemma 2.2(ii)]).

Theorem 4.2. Let $X$ be a uniformly convex and q-uniformly smooth Banach space with $q \in$ $(1,2]$. Assume that $A$ is a single-valued $v$-isa of order $q$ for some $v>0$ and $B$ is an $m$-accretive operator in $X$ such that $A+B$ is m-accretive. Assume that $S:=(A+B)^{-1}(0) \neq \emptyset$ and the conditions $(i)$ and (ii) of Theorem 4.1 are satisfied. Then the sequence $\left\{x_{n}\right\}$ generated by the split VAM (4.3) converges in norm to $Q(u)$, where $Q$ is the sunny nonexpansive retraction from $\Pi_{X}$ onto $S$.

Proof. Set $T_{n}:=T_{\lambda_{n}}=J_{\lambda_{n}}^{B}\left(I-\lambda_{n} A\right)$. Then, by Lemma 4.1, Fix $\left(T_{n}\right)=S$ for all $n$. In addition, we may rewrite $x_{n+1}$ as

$$
x_{n+1}=\alpha_{n} f\left(x_{n}\right)+\left(1-\alpha_{n}\right) T_{n} x_{n} .
$$

We observe that $\left\{x_{n}\right\}$ is bounded (so that Lemma 4.2 is applicable). As a matter of fact, Eq. (3.5) still holds for the sequence $\left\{x_{n}\right\}$ defined by (4.5), which can be obtained by replacing $J_{\lambda_{n}}^{A}$ with $T_{n}$ in the proof of (3.5). It then turns out that $\left\|x_{n+1}-T_{n} x_{n}\right\| \rightarrow 0$. From (4.5), we have $\left\|x_{n+1}-T_{n} x_{n}\right\|=\alpha_{n}\left\|f\left(x_{n}\right)-T_{n} x_{n}\right\| \leq M \alpha_{n} \rightarrow 0$, where $M$ is a constant such that

$$
M \geq \max \left\{\left\|T_{\lambda_{n}} x_{n}-x_{n}\right\|,\left\|T_{\lambda_{n}} x_{n}-f\left(x_{n}\right)\right\|\right\}, \quad n \geq 0 .
$$


Let $\tilde{x}=Q(f)$. Using Lemma 4.2, we conclude that

$$
\begin{aligned}
\left\|x_{n+1}-\tilde{x}\right\|^{q}= & \left\|\left(1-\alpha_{n}\right)\left(T_{n} x_{n}-\tilde{x}\right)+\alpha_{n}\left(f\left(x_{n}\right)-\tilde{x}\right)\right\|^{q} \\
\leq & \left(1-\alpha_{n}\right)^{q}\left\|T_{n} x_{n}-\tilde{x}\right\|^{q}+q \alpha_{n}\left\langle f\left(x_{n}\right)-\tilde{x}, J_{q}\left(x_{n+1}-\tilde{x}\right)\right\rangle \\
\leq & \left(1-\alpha_{n}\right)^{q}\left\{\left\|x_{n}-\tilde{x}\right\|^{q}-\lambda_{n}\left(v q-\lambda_{n}^{q-1} \kappa_{q}\right)\left\|A x_{n}-A \tilde{x}\right\|^{q}\right. \\
& \left.-\phi\left(\left\|x_{n}-\lambda_{n} A x_{n}+T_{n} x_{n}+\lambda_{n} A \tilde{x}\right\|\right)\right\} \\
& +q \alpha_{n}\left(\left\langle f\left(x_{n}\right)-f(\tilde{x}), J_{q}\left(x_{n+1}-\tilde{x}\right)\right\rangle+\left\langle f(\tilde{x})-\tilde{x}, J_{q}\left(x_{n+1}-\tilde{x}\right)\right\rangle\right) .
\end{aligned}
$$

By conditions (i)-(ii), we may assume that

$$
\left(1-\alpha_{n}\right)^{q} \geq \sigma \quad \text { and } \quad \lambda_{n}\left(v q-\lambda_{n}^{q-1} \kappa_{q}\right) \geq \sigma
$$

for all $n$ and some $0<\sigma<1$. Also using Young's inequality (i.e., $a b \leq a^{q} / q+b^{q^{\prime}} / q^{\prime}$, where $a, b>0$ and $q^{\prime}=q /(q-1)$ ), we obtain (recall that $f$ is an $\alpha$-contraction)

$$
\begin{aligned}
\left\langle f\left(x_{n}\right)-f(\tilde{x}), J_{q}\left(x_{n+1}-\tilde{x}\right)\right\rangle & \leq \alpha\left\|x_{n}-\tilde{x}\right\| \cdot\left\|x_{n+1}-\tilde{x}\right\|^{q-1} \\
& \leq \alpha\left(\frac{1}{q}\left\|x_{n}-\tilde{x}\right\|^{q}+\frac{q-1}{q}\left\|x_{n+1}-\tilde{x}\right\|^{q}\right) .
\end{aligned}
$$

From (4.7), we arrive at

$$
\begin{aligned}
\left\|x_{n+1}-\tilde{x}\right\|^{q} \leq & \left(1-\alpha_{n}\right)^{q}\left\|x_{n}-\tilde{x}\right\|^{q}-\sigma\left[\left\|A x_{n}-A \tilde{x}\right\|^{q}+\phi\left(\left\|x_{n}-\lambda_{n} A x_{n}+T_{n} x_{n}+\lambda_{n} A \tilde{x}\right\|\right)\right] \\
& \left.+\alpha \alpha_{n}\left(\left\|x_{n}-\tilde{x}\right\|^{q}+(q-1)\left\|x_{n+1}-\tilde{x}\right\|^{q}\right)+q \alpha_{n}\left\langle f(\tilde{x})-\tilde{x}, J_{q}\left(x_{n+1}-\tilde{x}\right)\right\rangle\right) .
\end{aligned}
$$

Solving for $\left\|x_{n+1}-\tilde{x}\right\|^{q}$ yields

$$
\begin{aligned}
\left\|x_{n+1}-\tilde{x}\right\|^{q} \leq & \frac{\left(1-\alpha_{n}\right)^{q}+\alpha \alpha_{n}}{1-\alpha(q-1) \alpha_{n}}\left\|x_{n}-\tilde{x}\right\|^{q} \\
& -\frac{\sigma}{1-\alpha(q-1) \alpha_{n}}\left[\left\|A x_{n}-A \tilde{x}\right\|^{q}+\phi\left(\left\|x_{n}-\lambda_{n} A x_{n}+T_{n} x_{n}+\lambda_{n} A \tilde{x}\right\|\right)\right] \\
& \left.+\frac{q \alpha_{n}}{1-\alpha(q-1) \alpha_{n}}\left\langle f(\tilde{x})-\tilde{x}, J_{q}\left(x_{n+1}-\tilde{x}\right)\right\rangle\right) .
\end{aligned}
$$

Introducing

$$
\beta_{n}:=1-\frac{\left(1-\alpha_{n}\right)^{q}+\alpha \alpha_{n}}{1-\alpha(q-1) \alpha_{n}}=\frac{1-q \alpha \alpha_{n}-\left(1-\alpha_{n}\right)^{q}}{1-\alpha(q-1) \alpha_{n}} \rightarrow 0\left(\text { as } \alpha_{n} \rightarrow 0\right),
$$

we may rewrite (4.8) as

$$
\left\|x_{n+1}-\tilde{x}\right\|^{q} \leq\left(1-\beta_{n}\right)\left\|x_{n}-\tilde{x}\right\|^{q}+\beta_{n} \gamma_{n},
$$

where

$$
\begin{aligned}
\gamma_{n}:= & \frac{q \alpha_{n}}{1-q \alpha \alpha_{n}-\left(1-\alpha_{n}\right)^{q}}\left\langle f(\tilde{x})-\tilde{x}, J_{q}\left(x_{n+1}-\tilde{x}\right)\right\rangle \\
& -\frac{\sigma}{1-q \alpha \alpha_{n}-\left(1-\alpha_{n}\right)^{q}}\left[\left\|A x_{n}-A \tilde{x}\right\|^{q}+\phi\left(\left\|x_{n}-\lambda_{n} A x_{n}+T_{n} x_{n}+\lambda_{n} A \tilde{x}\right\|\right)\right] .
\end{aligned}
$$

It is not hard to find that

$$
\lim _{n \rightarrow \infty} \frac{\beta_{n}}{\alpha_{n}}=q \quad \text { and } \quad \lim _{n \rightarrow \infty} \frac{1-q \alpha \alpha_{n}-\left(1-\alpha_{n}\right) q}{\alpha_{n}}=q(1-\alpha)>0 .
$$

Consequently, we have $\sum_{n=0}^{\infty} \beta_{n}=\infty$ by virtue of condition (i). 
Since $\left\{x_{n}\right\}$ is bounded, we have that $\left\{\gamma_{n}\right\}$ is bounded as well. Let $\left\{x_{n_{k}}\right\}$ be a subsequence of $\left\{x_{n}\right\}$ such that

$$
\limsup _{n \rightarrow \infty} \gamma_{n}=\lim _{k \rightarrow \infty} \gamma_{n_{k}}
$$

With no loss of generality, we may also assume that $x_{n_{k}} \rightarrow \hat{x}$ weakly and $\lim _{k \rightarrow \infty}\langle f(\tilde{x})-$ $\left.\tilde{x}, J_{q}\left(x_{n_{k}+1}-\tilde{x}\right)\right\rangle$ exists. We then assert that

$$
\lim _{k \rightarrow \infty} \frac{\sigma}{1-q \alpha \alpha_{n_{k}}-\left(1-\alpha_{n_{k}}\right)^{q}}\left[\left\|A x_{n_{k}}-A \tilde{x}\right\|^{q}+\phi\left(\left\|x_{n_{k}}-\lambda_{n_{k}} A x_{n_{k}}-T_{n_{k}} x_{n_{k}}+\lambda_{n_{k}} A \tilde{x}\right\|\right)\right]
$$

exists. Since $\left(1-q \alpha \alpha_{n_{k}}-\left(1-\alpha_{n_{k}}\right)^{q}\right) \rightarrow 0$ (for $\alpha_{n_{k}} \rightarrow 0$ ), we must have

$$
\lim _{k \rightarrow \infty}\left[\left\|A x_{n_{k}}-A \tilde{x}\right\|^{q}+\phi\left(\left\|x_{n_{k}}-\lambda_{n_{k}} A x_{n_{k}}-T_{n_{k}} x_{n_{k}}+\lambda_{n_{k}} A \tilde{x}\right\|\right)\right]=0 .
$$

Consequently, we obtain that

$$
A x_{n_{k}} \rightarrow A \tilde{x} \quad \text { and } \quad x_{n_{k}}-T_{n_{k}} x_{n_{k}} \rightarrow 0 \quad \text { (both in norm). }
$$

Hence,

$$
\begin{aligned}
\left\|x_{n_{k}+1}-x_{n_{k}}\right\| & \leq \alpha_{n_{k}}\left\|f\left(x_{n_{k}}\right)-x_{n_{k}}\right\|+\left(1-\alpha_{n_{k}}\right)\left\|x_{n_{k}}-T_{n_{k}} x_{n_{k}}\right\| \\
& \leq M \alpha_{n_{k}}+\left\|x_{n_{k}}-T_{n_{k}} x_{n_{k}}\right\| \rightarrow 0 .
\end{aligned}
$$

We claim that $\left\{x_{n_{k}}\right\}$ is an approximating fixed point of $T$. This is because, by Lemma 4.1, for any $0<\lambda<\inf _{n} \lambda_{n}$ small enough, we have

$$
\left\|x_{n_{k}}-T_{\lambda} x_{n_{k}}\right\| \leq 2\left\|x_{n_{k}}-T_{\lambda_{n_{k}}} x_{n_{k}}\right\| \rightarrow 0
$$

Now we can apply Lemma 3.1 to see

$$
\limsup _{n \rightarrow \infty}\left\langle f(\tilde{x})-\tilde{x}, J\left(x_{n_{k}}-\tilde{x}\right)\right\rangle \leq 0 .
$$

This evidently implies that (recall that $J_{q}(x)=\|x\|^{q-2} J(x)$ for $x \neq 0$ )

$$
\limsup _{n \rightarrow \infty}\left\langle f(\tilde{x})-\tilde{x}, J_{q}\left(x_{n_{k}}-\tilde{x}\right)\right\rangle \leq 0 .
$$

Therefore,

$$
\begin{aligned}
\limsup _{n \rightarrow \infty} \gamma_{n} & =\lim _{k \rightarrow \infty} \gamma_{n_{k}} \\
& \leq \limsup _{k \rightarrow \infty} \frac{q \alpha_{n_{k}}}{1-q \alpha \alpha_{n_{k}}-\left(1-\alpha_{n_{k}}\right)^{q}}\left\langle f(\tilde{x})-\tilde{x}, J_{q}\left(x_{n_{k}+1}-\tilde{x}\right)\right\rangle \\
& =\limsup _{k \rightarrow \infty} \frac{1}{1-\alpha}\left\langle f(\tilde{x})-\tilde{x}, J_{q}\left(x_{n_{k}}-\tilde{x}\right)\right\rangle \\
& \leq 0 .
\end{aligned}
$$

Finally, applying Lemma 2.2 to (4.9) (i.e., we take $s_{n}:=\left\|x_{n}-\tilde{x}\right\|^{q}, \lambda_{n}:=\beta_{n}, \mu_{n}:=\gamma_{n}$, and $\sigma_{n} \equiv 0$ in Eq. (2.2)), we obtain $\left\|x_{n}-\tilde{x}\right\|^{q} \rightarrow 0$, namely, $x_{n} \rightarrow \tilde{x}$ in norm. The proof is complete.

Remark 4.1. The proof of Theorem 4.1 given in [21] depends heavily on Maingé's technical lemma (i.e., [26, Lemma 3.1]). Our Theorem 4.2 improves Theorem 4.1 twofold. The first is an extension to the viscosity approximation method for finding a zero of the sum of two accretive operators. This seems to be the first attempt in the literature. The second is that the proof we 
provided here avoided using Maingé's technical lemma (i.e., [26, Lemma 3.1]). Our proof is simpler and more straightforward.

\section{CONCLUSION}

We extended the viscosity approximation method (VAM) to accretive operators in a Banach space $X$ to find a zero of an $m$-accretive operator $A$ and of the sum of two $m$-accretive operators $A$ and $B$. We proved the strong convergence of the VAM algorithms (3.3) and (4.3) in the framework of the underlying space being uniformly convex and/or uniformly smooth (uniform Gâteaux differentiability is sufficient in some circumstances) without assuming weak continuity of the normalized and generalized duality maps. Our proof method is simple and avoids using Maingé's technical lemma (i.e., [26, Lemma 3.1]) that has recently been used in proving the strong convergence of some iterative methods for fixed point and optimization problems. The proof of this paper shows that it is unnecessary to employ this technical lemma in many situations.

In [1, Theorem 5], Reich also proved that, in a uniformly convex and uniformly smooth Banach space $X$, if $A$ is an $m$-accretive operator with zeros, then, for each $x \in X$, the strong $\lim _{\lambda \rightarrow 0} J_{\lambda}^{A} x$ exists and defines the unique sunny nonexpansive retraction from $X$ onto $\overline{D(A)}$. It is interesting to know whether the viscosity approximation method works out for the resolvents $J_{\lambda}^{A}$ as $\lambda \rightarrow 0$. Note that the results presented in Sections 3 and 4 assume that $\left\{\lambda_{n}\right\}$ is bounded from below away from zero and the methods may fail to work for the case that $\lambda$ may approach to zero.

\section{Acknowledgments}

The authors extend their appreciation to the Deanship of Scientific Research at King Saud University for funding this work through research group no (RG-1440-058).

\section{REFERENCES}

[1] S. Reich, Strong convergence theorems for resolvents of accretive operators in Banach spaces, J. Math. Anal. Appl. 75 (1980), 287-292.

[2] W.R. Mann, Mean value methods in iteration, Proc. Amer. Math. Soc. 4 (1953), 506-510.

[3] B. Halpern, Fixed points of nonexpanding maps, Bull. Amer. Math. Soc. 73 (1967) 957-961.

[4] G. Lopez, V. Martin, H.K. Xu, Halpern's iteration for nonexpansive mappings, in "Nonlinear Analysis and Optimization I: Nonlinear Analysis," Contemp. Math. 513 (2010), 211-230.

[5] T. Dominguez Benavides, G. Lopez Acedo, H.K. Xu, Iterative solutions for zeros of accretive operators, Math. Nachr. 248-249 (2003), 62-71.

[6] J.G. O'Hara, P. Pillay, H.K. Xu, Iterative approaches to convex feasibility problems in Banach spaces, Nonlinear Anal. 64 (2006), 2022-2042.

[7] K. Aoyama, M. Toyoda, Approximation of zeros of accretive operators in a Banach space, Israel J. Math. 220 (2017), 803-816.

[8] H. Attouch, Viscosity approximation methods for minimization problems, SIAM J. Optim. 6 (1996), , 769806.

[9] A. Moudafi, Viscosity approximation methods for fixed-points problems, J. Math. Anal. Appl. 241 (2000), $46-55$.

[10] H.K. Xu, Viscosity approximation methods for nonexpansive mappings, J. Math. Anal. Appl. 298 (2004), 279-291.

[11] I. Cioranescu, Geometry of Banach Spaces, Duality Mappings and Nonlinear Problems, Kluwer Academic Publishers, Dordrecht, 1990. 
[12] F.E. Browder, Fixed point theorems for nonlinear semicontractive mappings in Banach spaces, Arch. Rational Mech. Anal. 21 (1966), 259-269.

[13] F.E. Browder, Nonlinear equations of evolution and nonlinear accretive operators in Banach spaces, Bull. Amer. Math. Soc. 73 (1967), 867-874.

[14] F.E. Browder, Nonlinear mappings of nonexpansive and accretive type in Banach spaces, Bull. Amer. Math. Soc. 73 (1967), 875-882.

[15] F.E. Browder, Convergence theorems for sequences of nonlinear operators in Banach spaces, Math. Z. 100 (1967), 201-225.

[16] F.E. Browder, Convergence of approximants to fixed points of nonexpansive non-linear mappings in Banach spaces, Arch. Rational Mech. Anal. 24 (1967). 82-90.

[17] H.K. Xu, T.H. Kim, Y. Yin, Weak continuity of the normalized duality map, J. Nonlinear Convex Anal. 15 (2014), 595-604.

[18] H.K. Xu, Inequalities in Banach spaces with applications, Nolinear Anal. 16 (1991), 1127-1138.

[19] S. Reich, Extesion problems for accretive sets in Banach spaces, J. Funct. Anal. 26 (1977), 378-395.

[20] K. Goebel, S. Reich, Uniform Convexity, Hyperbolic Geometry, and Nonexpansive Mappings, Marcel Dekker, New York, 1984.

[21] G. Lopez, V. Martin-Marquez, F. Wang, H.K. Xu, Forward-backward splitting methods for accretive operators in Banach spaces, Abst. Appl. Anal. 2012 (2012), Article ID 109236.

[22] V. Barbu, Nonlinear Differential Equations of Monotone Types in Banach Spaces, Springer, New York, 2010.

[23] H.K. Xu, Iterative algorithms for nonlinear operators, J. London Math. Soc. 66 (2002), 240-256.

[24] S. Saejung, P. Yotkaew, Approximation of zeros of inverse strongly monotone operators in Banach spaces, Nonlinear Anal. 75 (2012), 742-750.

[25] R.E. Bruck, S. Reich, Nonexpansive projections and resolvents of accretive operators in Banach spaces, Houston J. Math. 3 (1977), 459-470.

[26] P.E. Maingé, Strong convergence of projected subgradient methods for nonsmooth and nonstrictly convex minimization, Set-Valued Anal. 16 (2008), 899-912.

[27] P.L. Lions, B. Mercier, Splitting algorithms for the sum of two nonlinear operators, SIAM J. Numer. Anal. 16 (1979), 964-979.

[28] G.B. Passty, Ergodic convergence to a zero of the sum of monotone operators in Hilbets space, J. Math. Anal. Appl. 72 (1979), 383-390. 